\title{
REMARK ON THE DUALITY FOR NONCOMMUTATIVE COMPACT GROUPS
}

\author{
TADASI NAKAYAMA
}

The duality theorem for noncommutative compact groups was established by Tannaka, ${ }^{1}$ and later, but independently, by Krein. ${ }^{2}$ Different proofs have been given by Bochner and Yosida. ${ }^{3}$ The purpose of the present note is to supplement the theorem by a duality between (closed) subgroups and certain representation-theoretically defined systems. It provides also an extension of Tannaka's theorem itself. As a matter of fact, it is more or less known that the subgroups are in 1-1 correspondence with, say, left-invariant subrings with conjugation of the ring of coefficients of (irreducible) representations. ${ }^{4}$ But we propose to make the dual objects to be associated with subgroups free from the properties concerned with the group operation, such as left-translations, and obtain purely representationtheoretical dual systems. ${ }^{5}$ Our intention is also to have a formulation which gives directly the subgroups of a character group in the abelian case. Thus our work has no novelty in analytical respects, and our concern lies mainly in its formulation.

1. Let $G$ be a topological group. We take a representation $\mathfrak{D}_{k}$ from each equivalence class of irreducible bounded representations of $G$. Denote the totality of (finite) linear combinations of the elements of all the $\mathfrak{D}_{k}$ over the field $\Omega$ of complex numbers by $\Re$, and that of the elements in a fixed $\mathscr{D}_{k}$ by $\Re_{k}$. They are rings, here with respect to convolution, and indeed two-sided ideals in the ring $R$ of all almost periodic functions. They are also two-sided $G$-moduli, operations of $z \in G$ on a function $f(x)$ on $G$ being defined by $z \cdot f(x)$ $=f\left(z^{-1} x\right), f(x) \cdot z=f\left(x z^{-1}\right)$. Let $s_{k}$ be the degree of $\mathfrak{D}_{k}$. $\mathfrak{R}_{k}$ is directly de-

Received by the editors December 8, 1950.

${ }^{1} \mathrm{~T}$. Tannaka, Über den Duatitätssatz der nichtkommutativen topologischen Gruppen, Tôhoku Math. J. vol. 45 (1938).

2 M. Krein, On almost periodic functions on a topological group, C. R. (Doklady) Acad. Sci. URSS. vol. 30 (1941).

${ }^{3}$ S. Bochner, On a theorem of Tannaka and Krein, Ann. of Math. vol. 43 (1942); $\mathrm{K}$. Yosida, On the duality theorem of non-commutative compact groups, Proc. Imp. Acad. Tokyo vol. 19 (1943); cf. also C. Chevalley, Theory of Lie groups, I, Princeton, 1946, chap. VI.

${ }^{4}$ It does not seem to the writer, however, that this has been stated in the literature. Hence the following exposition contains necessarily some arguments, in this context, which are rather well known.

${ }^{5}$ Or, more precisely, systems which are defined in terms of the mutual relationship of representations only. 
composed into $s_{k}$ irreducible $G$ - (or $R$ - or $\Re$-) left-moduli. For instance, the $s_{k}$ columns of $\mathfrak{D}_{\kappa}(x)$ span such $s_{\kappa}$ left-moduli. Indeed $z \mathfrak{D}_{k}(x)$ $=\mathfrak{D}_{\kappa}\left(z^{-1}\right) \mathfrak{D}_{\kappa}(x)$. As our algebraic preliminary we note the following lemma. ${ }^{6}$

LEMMA 1. If $\mathrm{t}(x)$ is a column in $\Re$ (or in $R$ ) such that at $(x)$ $=\mathfrak{D}_{k}\left(z^{-1}\right) \mathfrak{t}(x)(z \in G)$, then $\mathfrak{t}(x)$ is a linear combination of $s_{k}$ columns of $\mathfrak{D}_{k}(x)$.

Now, let $H$ be a closed subgroup in $G$. The set of all $f$ in $R$ with $f(x a)=f(x)$ for every $a \in H$ forms a subring $R^{\prime}$ of $R$; such an $f$ can be considered as a function on the space of right cosets $G \bmod H$, and conversely. $\Re^{\prime}=\Re \cap R^{\prime}$ is a subring of $\Re$. $R^{\prime}, \Re^{\prime}$ are $G$ - (and $R-$ ) leftallowable. The same is the case for $\Re_{\alpha}^{\prime}=\Re_{k} \cap R^{\prime}$, and $\Re^{\prime}$ is the direct sum $\sum_{\kappa} \Re_{\kappa}^{\prime}$. Indeed each $f \in \Re^{\prime}$ is the (essentially finite) sum of $e_{\kappa} \times f \in \Re_{k}^{\prime}, e_{k}$ being the unit of $\Re_{\kappa}$.

Lemma 2. Let $G$ be maximally almost periodic. If an element a in $G$ satisfies $f(x a)=f(x)$ for every $f(x)$ in $\Re^{\prime}$, then $a \in H$.

This follows readily from the completeness of $\Re^{\prime}$ in $R^{\prime}$ if we observe that $R^{\prime}$ is the set of harmonics on the homogeneous space $G$ mod $H ;^{7}$ further, we have the following lemma.

LEMma 3. If a linear function $X$ on the linear space $R^{\prime}$ satisfies $X(f \bar{f}) \geqq 0$ (for every $\left.f \in \Re^{\prime}\right)$, then $X(f) \geqq 0$ for every $f$ in $\Re^{\prime}$ with $f \geqq 0$.

The proof runs almost in the same manner as in the proofs of Bochner and Krein for the case $H=1, \Re^{\prime}=\Re$.

2. Now $\Re_{\kappa}^{\prime}=\Re_{\kappa} \cap R^{\prime}$ is a direct sum of a certain number, say $t_{\kappa}$, of irreducible $G$-left-moduli. Take their bases in column forms transforming according to $\mathfrak{D}_{k}\left(z^{-1}\right)$, and denote the matrix composed of them by $\mathfrak{T}_{\kappa}(x) ; z \mathfrak{T}_{\kappa}(x)=\mathfrak{D}_{\kappa}\left(z^{-1}\right) \mathfrak{T}_{\kappa}(x)$. By Lemma 1 there is a matrix $T_{k}$ of type $\left(s_{k}, t_{k}\right)$ in $\Omega$ satisfying

$$
\mathfrak{T}_{\kappa}(x)=\mathfrak{D}_{\kappa}(x) T_{\kappa} .
$$

(Here $\Re^{\prime}$ is the left ideal of $R$ induced by the (left) ideal $1 \Omega$ (1 being the function identically equal to 1 ) of the ring of almost periodic functions on $H$, and our multiplicity $t_{k}$ of $\mathfrak{D}_{k}$ in the representation of

${ }^{6}$ This is, in fact, a particular instance of a general theorem on principal left ideals generated by idempotent elements (combined with a bit of the theory of almost periodic functions).

${ }^{7} \mathrm{H}$. Weyl, Harmonics on homogeneous manifolds, Ann. of Math. vol. 35 (1934); cf. also A. Weil, L'intégration dans les groupes topologiques et ses applications, Actualités Scientifiques et Industrielles, 1940, chap. V. 
$G$ defined by its $\mathfrak{D}_{k}$-component $\Re_{k}^{\prime}$ is equal to the multiplicity of the unit representation in the restriction of $\mathfrak{D}_{\mathrm{K}}$ in $\left.H.\right)^{8}$ It follows from the multiplication property of $\mathfrak{D}_{\mathfrak{k}}(x)$ that Lemma 2 may be restated in the following form.

LEMMA 4. $H$ consists of the totality of elements $a$ in $G$ such that $\mathfrak{T}_{k}(a)$ $=T_{k}$ for every $\mathrm{k}$.

The Kronecker product $\mathfrak{D}_{\kappa}(x) \times \mathfrak{D}_{\lambda}(x)$ is decomposed into $\mathfrak{D}_{\mu}(x)$ :

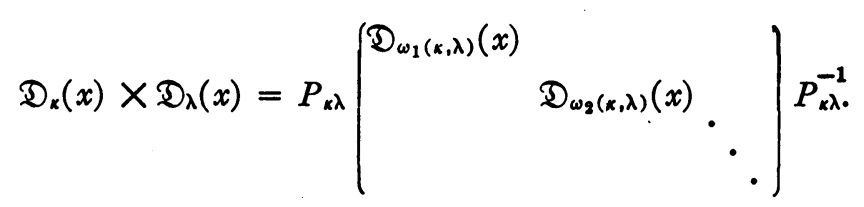

Hence

$$
\begin{aligned}
& \mathfrak{T}_{k}(x) \times \mathfrak{I}_{\lambda}(x)=\left(\mathfrak{D}_{k}(x) \times \mathfrak{D}_{\lambda}(x)\right)\left(T_{k} \times T_{\lambda}\right) \\
& =P_{k \lambda}\left(\begin{array}{lll}
\mathfrak{D}_{\omega_{1}(\kappa, \lambda)}(x) & & \\
& \mathfrak{D}_{\omega_{2}(\kappa, \lambda)}(x) & \\
& & \ddots
\end{array}\right) P_{k \lambda}^{-1}\left(T_{\alpha} \times T_{\lambda}\right) .
\end{aligned}
$$

The elements of the left side, whence those of $P_{k \lambda}^{-1}\left(\mathfrak{T}_{k}(x) \times \mathfrak{T}_{\lambda}(x)\right)$, belong to $\Re^{\prime}$. By expressing $\Re_{k}$ as a direct sum of $\Re_{k}^{\prime}$ and a complementary left ideal, we see easily that

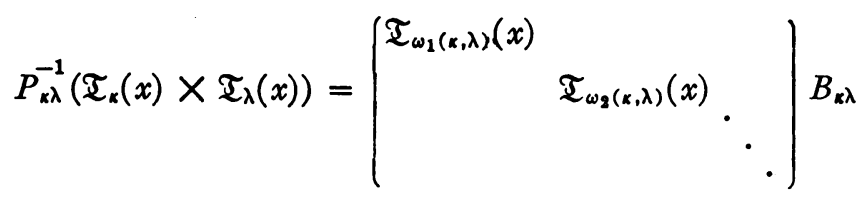

with a matrix $B_{k \lambda}$ of type $\left(\sum_{i} t_{\omega_{i}(\alpha, \lambda)}, t_{k} t_{\lambda}\right)$ in $\Omega$.

Further, $\overline{\mathfrak{D}}_{\boldsymbol{K}}(x)$ is equivalent to a certain $\mathfrak{D}_{\mu}(x)$ :

$$
\overline{\mathfrak{D}}_{\mu}(x)=U_{\kappa} \mathfrak{D}_{\mu(\kappa)}(x) U_{\kappa}^{-1} \text {. }
$$

Hence $U_{k}^{-1} \overline{\mathfrak{D}}_{k}(x)$ transforms according to $\mathfrak{D}_{\mu(x)}\left(z^{-1}\right)$. We can treat the matrix $U_{k}^{-1} \bar{D}_{k}(x) \bar{T}_{k}=U_{k}^{-1} \mathfrak{T}_{k}(x)$ similarly as above and obtain

$$
U_{k}^{-1} \widetilde{\mathfrak{T}}_{k}(x)=\mathfrak{T}_{\mu(k)}(x) C_{k}
$$

with a matrix $C_{k}$ of degree $t_{k}$ in $\Omega .\left({ }^{*}\right),\left({ }^{* *}\right)$ may be written, of course,

8 For the general relation, which generalizes Frobenius' theorem, see T. Nakayama, A remark on representations of groups, Bull. Amer, Math. Soc. vol. 44 (1938); cf. also A. Weil, loc. cit. p. 82. 
as

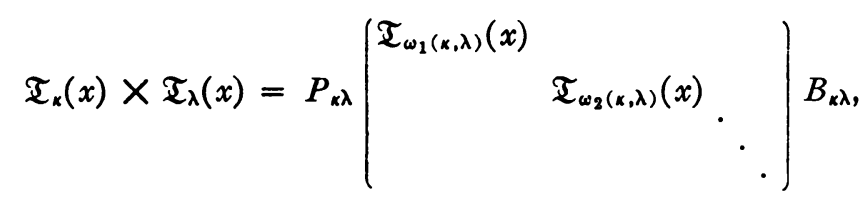

$$
\begin{aligned}
& \overline{\mathfrak{T}}_{\kappa}(x)=U_{k} \mathfrak{T}_{\mu(\kappa)}(x) C_{\kappa} .
\end{aligned}
$$

(1) shows that $\Re^{\prime}$ is also a ring in the sense of usual functionmultiplication (which was however evident from the beginning) and it prescribes the multiplication of the ring $\Re^{\prime}$ in the functional sense, that is, the function-ring $\Re^{\prime}$ as we shall call it. (2) describes the conjugation in $\Re^{\prime}$. We call $\left\{T_{\kappa} ; B_{k \lambda}, C_{k}\right\}$ a duality system belonging to the subgroup $H$ (under our fixed choice of $\omega_{i}(k, \lambda), P_{k \lambda}$, and $U_{k}$ ).

As far as we fixed our choice of $t_{k}$-component left ideals in $\Re_{k}^{\prime}$, each column in $T_{k}$ is determined up to nonzero factors (from $\Omega$ ), that is, $T_{\kappa}$ itself is determined up to (nonsingular) diagonal matrices as right factor. With a different choice of components we have to deal with $T_{k} W_{\kappa}$ with general nonsingular $W_{\kappa}$ (of degree $t_{\kappa}$ ). Then $B_{\kappa \lambda}, C_{\kappa}$ will be replaced by

$$
\left(W_{\omega_{1}(\kappa, \lambda)} \cdot \int^{-1} B_{\kappa \lambda}\left(W_{\kappa} \times W_{\lambda}\right), \quad W_{\mu(\kappa)}^{-1} C_{\kappa} \bar{W}_{\kappa} .\right.
$$

So we consider the system

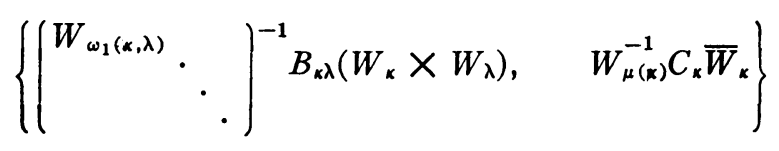

as equivalent to our original one. We may also analyze a different choice of $\omega_{i}(\kappa, \lambda), P_{\kappa \lambda}$, and $U_{\kappa}$. However, we want rather to fix them once and for all, since they are concerned only with our total group (or, rather, with its representations).

3. Conversely let us consider a system $\left\{T_{k} ; B_{k \lambda}, C_{k}\right\}$ of matrices $T_{k}$ of respective type $\left(s_{k}, t_{k}\right)$ and rank $t_{k}$, and matrices $B_{k \lambda}, C_{k}$ of respective type $\left(\sum_{i} t_{\omega_{i}(\kappa \lambda)}, t_{k} t_{\lambda}\right),\left(t_{k}, t_{\kappa}\right)$, in $\Omega$, such that (1), (2) hold for $\mathfrak{T}_{\kappa}(x)=\mathfrak{D}_{\kappa}(x) T, t_{\kappa}$ being arbitrary (non-negative) integers $\left(\leqq s_{\kappa}\right)$. (The assumption on the rank of $T_{k}$ is not essential; it is merely to eliminate dummy columns.) We call such a system a duality system (proper, without referring to a subgroup).

Given such a duality system, let 


$$
H=\left\{a \mid T_{\kappa}=\mathfrak{T}_{\kappa}(a)=\mathfrak{D}_{\kappa}(a) T_{\kappa}\right\} .
$$

Clearly $H$ is a closed subgroup; this in fact has nothing to do with $B_{k \lambda}, C_{\kappa}$. For, if $a, b \in H$, then $\mathfrak{D}_{\kappa}(a b) T_{\kappa}=\mathfrak{D}_{\kappa}(a) \mathfrak{D}_{\kappa}(b) T_{\kappa}=\mathfrak{D}_{\kappa}(a) T_{\kappa}=T_{\kappa}$, and $\mathfrak{D}_{\kappa}\left(a^{-1}\right) T_{\kappa}=\mathfrak{D}_{k}(a)^{-1} T_{\kappa}=\mathfrak{D}_{k}(a)^{-1} \mathfrak{D}_{k}(a) T_{k}=T_{k}$, proving that $H$ is a subgroup. Its closedness is evident. Further, if $a \in H$ we have

$$
\mathfrak{T}_{\kappa}(x a)=\mathfrak{D}_{\kappa}(x a) T_{\kappa}=\mathfrak{D}_{\kappa}(x) \mathfrak{D}_{\kappa}(a) T_{\kappa}=\mathfrak{D}_{\kappa}(x) T_{\kappa}=\mathfrak{T}_{\kappa}(x)
$$

for any $x \in G$.

LEMMA 5. Linear combinations of coefficients of $\mathfrak{T}_{k}(x)$ form indeed the totality of functions $f(x)$ in $\Re$ satisfying $f(x a)=f(x)(a \in H)$.

We first observe that with any fixed $x_{0} \in G$ our subgroup $H$ is characterized also as the totality of $a \in G$ such that $\mathfrak{T}_{k}\left(x_{0} a\right)=\mathfrak{T}_{k}\left(x_{0}\right)$. Then the lemma follows by the well known theorem on rings, with conjugation, of sufficiently many continuous functions. ${ }^{9}$ Namely, the closure in $R$ of our module, spanned by the elements of $\mathfrak{T}_{k}(x)$, forms, according to our remark and the relations (1), (2), such a ring on the space $G \bmod H$, whence on its compact cover. Thus the closed module coincides with the totality of continuous functions on the compact cover of $G \bmod H$. Since our original module is closed in $\Re$, as follows, for instance, from the orthogonality of coefficients of $\mathfrak{D}_{\kappa}$, this implies our assertion.

From our lemma follows readily that our given system is really a duality system belonging to $H$. On combining this with the observation (Lemma 4 , in particular) in the preceding section, we can assert the following theorem.

THEOREM 1. Closed subgroups of a maximally almost periodic group are in 1-1 dual correspondence with equivalence classes of duality systems in $G$.

It is perhaps needless to say that the statement provides also a generalization of Van Kampen's theorem on a system of sufficiently many representations.

4. From the analytically fundamental Lemma 3, we obtain the following lemma.

Lemma 6. Let $G$ be compact. For a 1-dimensional representation of the function-ring $\Re^{\prime}$ which preserves the conjugation there is an element $x$ in $G$ such that $f \rightarrow f(x)$ is really the representation.

\footnotetext{
${ }^{9}$ See I. Gelfand-G. Silov, Verschiedene Methoden der Einfïhrung der Topologie in die Menge der maximalen Ideale eines normierten Ringes, Rec. Math. (Mat. Sbornik) N.S. vol. 9 (1941).
} 
For, such a representation $f \rightarrow X(f)$ certainly satisfies $X(f \bar{f}) \geqq 0$. Hence $X(f) \geqq 0$ whenever $f \geqq 0\left(f \in \Re^{\prime}\right)$. It follows then readily that $X$ is continuous, and can be extended to a 1-dimensional continuous representation of $R^{\prime}$ (preserving conjugation). But $R^{\prime}$ is nothing but the whole ring of continuous functions on $G \bmod H$, and its 1-dimensional continuous representation can be given by a point, that is, by an element $x$ of $G \bmod H$.

Suppose now that there are associated to $\mathfrak{T}_{k}(x)$ matrices $\mathfrak{T}_{k}(X)$ in $\Omega$ of respective type $\left(s_{k}, t_{k}\right)$ such that

$$
T_{\kappa}(X) \times T_{\kappa}(X)=P_{\kappa \lambda}\left(\begin{array}{ll}
T_{\omega_{1}(\kappa, \lambda)}(X) & \\
& T_{\omega_{2}(\kappa, \lambda)}(X) \cdot \\
& \\
&
\end{array}\right) B_{\kappa \lambda,}
$$

$\left\{T_{k} ; B_{k \lambda}, C_{k}\right\}$ being a duality system. Then $X$ gives a 1-dimensional representation, preserving conjugation, of the function-ring. Hence we have the following theorem.

Theorem 2. If $G$ is compact, there exists an element $x$ in $G$ such that $\mathfrak{T}_{k}(X)=\mathfrak{T}_{k}(x)$.

This provides an extension of Tannaka's theorem itself, again in the algebraic respect. It is of course easy to show that the weak topology topologizes the set of $X$ so as to make it homeomorphic to the space $G$ mod the subgroup $H$ belonging to our duality system.

In closing, the writer wants to repeat the point that his duality systems are defined purely in terms of representations, or their behavior with respect to the Kronecker product, to be more precise, without appealing to the group and its group operation. If in particular $G$ is abelian, then of course $\mathfrak{D}_{k}$ are all 1-dimensional, and $T_{k}$ are simply numbers in $\Omega$. Under normalization with respect to our equivalence transformation, observed at the end of $\$ 2$, they are either 0 or 1 , and (1), (2) mean that those $\mathfrak{D}_{k}$ with $T_{k}=1$ form a subgroup of the character group. The writer assumes further that his formulation is suited for a Lie algebra version such as was given recently by HarishChandra ${ }^{10}$ for the whole group duality.

NAGOYA UNIVERSITY

10 Harish-Chandra, Lie algebras and the Tannaka duality theorem, Ann. of Math. vol. 51 (1950). 